ISSN: 0212-0267

DOI: http: //dx.doi.org/ro.I420I/hedu201938181194

\title{
EL CONCEPTO DE INFANCIA EN LA BAJA EDAD MEDIA EN CASTILLA A TRAVÉS DE LOS «ESPEJOS DE PRÍNCIPES» (SIGLOS XIII-XV)
}

\section{The concept of childhood in Late Middle Ages in Castile through the "Mirror for Princes" (I3th-Isth centuries)}

Enrique Asenjo Travesí

Universidad Complutense de Madrid

Correo-e: easenjotravesi@educa.madrid.org

Recepción: 3 de mayo de 20I8. Envío a informantes: ro de mayo de 2018 Aceptación definitiva: 12 de febrero de 2019

Resumen: Durante la Baja Edad Media, Castilla vive un resurgir de la literatura sapiencial en lo tocante a la formación moral e intelectual de los príncipes. Es este un fenómeno novedoso al calor del resurgir de la cultura clásica y la revisión de los textos latinos bajo una nueva dimensión en esta época. Los reinos bajomedievales hispanos adoptaron una nueva visión de los textos clásicos no solo desde el punto de vista moralizante cristiano, sino desde una óptica laica y más práctica. La literatura sapiencial o los «Espejos de Príncipes» constituyen un ejemplo del afán por formar al buen príncipe o monarca ante un contexto político cambiante y novedoso, pero también, como un recurso habitual en la Edad Media, se hace mirando al pasado clásico como referente inicial.

Palabras clave: Baja Edad Media; Castilla; literatura sapiencial; Espejo de Príncipes; cultura clásica; moral; intelectual.

Aвstract: During Late Middle Ages, we can notice a revive of the sapiential literature in order to teach Castilian princes in moral and practical knowledge about government. This is a phenomena that happens around a renewal of the Classic 
culture and the new interpretation of its meaning. That new point of view that appeared in the Late Medieval Spanish kingdoms tried to provide the throne heirs with new skills not only as moral examples, but with practical knowledge. The sapiential literature in the «Mirror for Princes» tries to teach the future kings or rulers in a changing context in politics, but as tipically in Middle Ages, also tries to face the future with a look in the past.

KeY words: Late Middle Ages; Castile; sapiential literature; Mirror for Princes; Classic culture; moral; intellectual.

\section{Introducción}

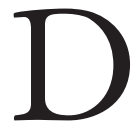

uRante la Edad Media la fe cristiana constituyó el elemento cultural más destacado de la vida de los europeos. La educación en la misma se desarrolló a través de los catecúmenos desde el siglo in d. C. Sin embargo, los objetivos de la educación cristiana y pagana eran diferentes. La primera buscaba hacer fieles seguidores de Dios, mientras que la segunda pretendía formar buenos ciudadanos. El objetivo de la pedagogía en época medieval consistía en restituir los bienes espirituales dañados por el pecado original, y confiaba la reparación humana de este mal a través del entendimiento, la virtud y la gracia divina $^{\mathrm{I}}$. Los valores más destacados dentro del mundo del conocimiento y la educación medieval fueron el entendimiento y la memoria. En cuanto a los centros de formación, destacaron sobre todo las escuelas monásticas medievales y, a partir del siglo xI, los estudios mayores y universidades. Durante la Baja Edad Media, la mayor parte de las principales ciudades desarrollaron universidades o estudios generales, que realizaron una labor docente que excedió del ámbito puramente eclesiástico. Por otro lado, la nobleza y el entorno cortesano mantuvieron un estilo formativo propio.

Hemos de analizar la visión que en la Edad Media se tenía tanto de los jóvenes como de la descendencia dentro de los núcleos familiares. En líneas generales, ambos han sido entendidos durante gran parte de la historia como un don por parte de las sociedades patriarcales. La abundancia de hijos fue tenida en gran medida como una riqueza y una fuente de bienes para las familias, al tiempo que la ausencia de la misma era tomada como una lacra, especialmente para las mujeres que no conseguían perpetuar la línea sucesoria. Incluso mujeres de elevada alcurnia del último siglo medieval gozaron de una mala prensa por este hecho, como le sucedió a la mujer de Alfonso V de Aragón, la reina doña María de Castilla².

Esta importancia que se manifiesta para unos aspectos contrasta con la crueldad con que los niños podían ser tratados en otros ámbitos cotidianos de las

Casado Rigalt, D.: Historia de la educación durante la Antigüedad y la Edad Media, Madrid, UDIMA, 20I5, p. 76.

2 Parece ser que tuvo graves desarreglos ginecológicos desde su juventud, teniendo su primera regla dos años después de casada. Giménez Soler, A.: «Retrato histórico de la reina doña María», Boletín de la Real Academia de Buenas Letras de Barcelona, I90I, pp. 72-8I. 
EL CONCEPTO DE INFANCIA EN LA BAJA EDAD MEDIA EN CASTILLA A TRAVÉS

DE LOS «ESPEJOS DE PRÍNCIPES» (SIGLOS XIII-XV)

ENRIQUE ASENJO TRAVESÍ

familias más desfavorecidas³. Las obras de literatura sapiencial de la Baja Edad Media nos dejan un reflejo del complejo mundo en el que se desarrollaron los niños de un estrato privilegiado, el de la nobleza y realeza, y en que quedaron reflejados los principios morales que se pretendía que los niños y, sobre todo, jóvenes, aprendiesen por mímesis e imitación de los adultos. Sin embargo, en otros estratos sociales, la visión de los niños como una pesada carga también continuó, tachándolos de inconstantes, olvidadizos, gritones, mentirosos, perezosos y llorones, entre otros calificativos ${ }^{4}$.

\section{La literatura de los Espejos de Príncipes en Castilla}

El momento a partir del cual el rey comienza a proyectar su imagen a los súbditos como un ejemplo sucede desde el siglo XI. Esta cuestión se une a la formación de un ideal de caballería medieval, en el que el príncipe, además de guerrero, es un buen cristiano. En esa medida, ese espejo sirve para proyectar la imagen propagandística del rey a la sociedad, pero también para que su heredero se observe a sí mismo en el reflejo mismo de ella, para que así imite fielmente los pasos de su predecesor. La rectitud moral del rey, así como su ejemplo, se constituyen en elementos vitales de cohesión y ordenación socials.

El caso de la Corona de Castilla no fue ajeno a esta nueva realidad, y en el siglo XIII, tanto los monarcas Fernando III como Alfonso X, como los posteriores de otras centurias, mostraron un creciente interés por la literatura especular como elemento de formación ${ }^{6}$. Para el caso de la Corona de Castilla, la primera obra de relevancia es el Libro de los doze sabios, redactado en época de Fernando III.

3 LANGer, W.: Historia de la infancia, Boston, 1973. «Posiblemente, el trato despiadado de los niños, desde la práctica del infanticidio y el abandono hasta la negligencia, los rigores de la envoltura en fajas, la inanición deliberada, las palizas, los encierros, etc., era y es simplemente un aspecto de la agresividad y crueldad que hay en el fondo de la naturaleza humana, de la indiferencia innata respecto de los derechos y sentimientos de los demás. Los niños, al ser físicamente incapaces de oponer resistencia a la agresión, eran víctimas de fuerzas sobre las cuales no tenían control y eran maltratados en muchas formas imaginables y en algunas casi inimaginables que expresaban los motivos conscientes o las más de las veces inconscientes de sus mayores». Prólogo.

4 Hay durísimos refranes que muestran un reflejo del imaginario colectivo de la época final de la Edad Media al respecto, como el francés ya del siglo xvi, que dice que «al pobre se le muere la vaca, y al rico el hijo»; FLANDRIN, J. L.: «Lugares comunes, tradicionales y modernos, sobre el niño en la familia», en La moral sexual en Occidente, Barcelona, ı984, p. 247; o el británico que reza: «Un hombre no debe confiar en una espada rota, ni en un necio, ni en un niño, ni en un fantasma, ni en un borracho»; García Herrero, M. C.: Las mujeres en Zaragoza en el siglo XV, Zaragoza, I99o, vol. I, p. 9I.

Nogales Rincón, D.: «Los espejos de príncipes en Castilla (siglos XIII-Xv): un ejemplo literario de la Castilla Bajomedieval», Medievalismo, I6 (2006), p. Iо.

6 Así, la figura del rey se proyectó con notable éxito entre la población letrada como un ingenio literario: durante el siglo xII ya aparecen notables ejemplos europeos como el de Juan de Salisbury, con su Policraticus, y ya en el xiII Gerardo de Cambray con el De principis instructione.

El Libro de los doze sabios posee un espacio propio dentro de la literatura sapiencial política, dado que no es un Speculum al uso. En primer lugar está redactado, si seguimos su prólogo, por doce sabios que dan consejos de buen gobierno al futuro rey, el infante Alfonso. Sin embargo, más 
Así, se tratan asuntos como la codicia, la fortaleza del rey, la castidad o la templanza, pero también la ira o la saña con la que el rey debe aplicarse contra quienes no guardan el servicio debido a Dios o por la tierra comunal ${ }^{8}$, etc. En general, se aprecia una agrupación de aspectos generales de lo que debe ser el conjunto de virtudes del buen rey en los primeros veinte capítulos, destacando valores tales como lealtad, justicia, templanza, amistad, generosidad, etc. Se observa también la crueldad como virtud con aquellos que son malos súbditos. El resto de los cánones tienen una aplicación mucho más concreta y práctica de las tareas de gobierno, mezclándose aspectos del ejercicio práctico de la guerra con el ejercicio de la justicia y administración del reino, e intercalando aspectos morales y consejos. En general, el infante, dentro de la particularidad que tiene la obra, que probablemente fue escrita total o parcialmente en tiempos del reinado de Alfonso $\mathrm{X}$ (aunque vaya dirigida a él), es tratado como un adulto en su formación.

Otra obra que entra dentro del género de la literatura sapiencial es el compendio de Flores de la Filosofía. Realmente se encuentra inserto dentro de varias recopilaciones de textos de literatura sapiencial y prosa variada castellana del siglo XIII, tanto de la Biblioteca Nacional de Madrid como de la Biblioteca de El Escorial. Por tanto, es un texto que nos ha llegado transcrito con posterioridad, aunque se calcula que fue redactado entre los reinados de Fernando III y Alfonso X el Sabio?. En general constituye un listado de virtudes y consejos para el buen gobernante, pero no deja entrever las características formativas del mismo, si no sólo los resultados de su formación. Se divide su organización interna en cinco grandes apartados, según Fernando Gómez Redondo ${ }^{\mathrm{IO}}$.

Cada apartado se centra en la aplicación y consecución de unos objetivos, pero no define el procedimiento. Además, mezclan elementos prácticos de las tareas de gobierno con otros que son más claramente moralizantes, muy típicos del mundo medieval, en los que el elemento religioso aparece bien definido. Las transcripciones realizadas sobre el texto original se han centrado más en la dimensión filológica que en el análisis e interpretación del concepto de buen príncipe o gobernante ${ }^{\mathrm{II}}$.

adelante nombra al infante como rey. Para algunos autores como Walsh el libro fue redactado en la corte de Fernando III; para otros como Bizzarri hay dos redacciones, una de época de Fernando III y otra rehecha a partir de la primera durante los primeros años del reinado de Alfonso X, para fortalecer su imagen frente a algunos de sus hermanos y la nobleza levantisca. Gómez REDONDo, F.: Historia de la prosa medieval castellana, vol. I, Madrid, I998, pp. 24I-246.

8 Walsh, J. K.: El libro de los doze sabios, Madrid, I975, p. 83.

9 En realidad, las fechas que se han barajado a lo largo del tiempo por los estudiosos son muy amplias, bailando entre el reinado de Alfonso VIII y el de Alfonso X. Gómez Redondo, F.: op. cit., p. 260.

1o Ibídem, p. 269. La primera comprende el primer capítulo (sobre cómo el hombre debe amar a Dios). La segunda (capítulos 2 a 9), el rey como centro de la vida de la corte. La tercera, (capítulos Io a 29), el hombre ante la vida de la corte, es la más amplia. La cuarta (capítulos 30 al 37) trata del «seso» como guía espiritual. La quinta se cierra con un capítulo sobre Dios y la salvación del alma.

"Lucía Megías, J. M.: Flores de filosofía, Transcripción semipaleográfica del ms. 9428 de la Biblioteca Nacional de Madrid (ff. I-I8), Seminario de Edición y Crítica Textual (I5-IO-I997), http:// parnaseo.uv.es/Memorabilia/Flores2.html. Óscar Bizzarri, H.: Flores de filosofía, Ms. escur. S.II.I3, Seminario de Edición y Crítica Textual (I5-IO-1997), http://parnaseo.uv.es/Memorabilia/Floresı.html. 
EL CONCEPTO DE INFANCIA EN LA BAJA EDAD MEDIA EN CASTILLA A TRAVÉS DE LOS «ESPEJOS DE PRÍNCIPES» (SIGLOS XIII-XV)

ENRIQUE ASENJO TRAVESÍ

Es frecuente encontrar también tratados relativos a la formación de príncipes que ponen a una figura de renombre histórico como exempla de buen o mal gobierno. El referente tradicional es Alejandro Magno, y sobre él tratan varios textos castellanos recogidos en diferentes versiones, que son el Secreto de los secretos y Poridat de las poridades ${ }^{12}$. En realidad, muestran la estructura de un manual de buen gobierno que refleja aspectos o consejos prácticos, sacados o referenciados en Alexandre, que es como denomina al monarca, en un intento de equipararle con un nuevo Alejandro Magno. Es un modo de mostrar al rey como un héroe y un guerrero victorioso, al tiempo que manifiesta su vinculación con el pasado clásico ${ }^{13}$.

La literatura sapiencial del regimiento de príncipes cambió a finales del siglo XIII con la obra de los Castigos del rey don Sancho. El objetivo era el mismo, mostrar al futuro rey Fernando IV el recto camino para formarse un juicio como buen monarca, pero el tono varió notablemente, con un marcado carácter teológico-religioso, en el que se procura mostrar al rey los errores de juicio que no debe cometer, y que para formarse como monarca primero debe tener una rectitud moral intachable como buen cristiano. Por lo tanto, el mundo espiritual del rey debe ser el rector de las acciones del monarca en la tierra, es decir, en su Reino. El juicio moral y religioso es la base del pensamiento y, por lo tanto, la formación del rey en el conocimiento profundo de los ejemplos de vida de los personajes bíblicos es la fundamentación de sus acciones y decisiones, según este largo texto ${ }^{\mathrm{I} 4}$. Los exempla que muestra son siempre protagonizados por personajes bíblicos, santos o padres de la Iglesia.

Existen múltiples fuentes tardomedievales sobre la educación de príncipes. Una de las más llamativas es la glosa castellana del Regimiento de Príncipes de Egidio Romano, llevada a cabo por Juan García de Castrojeriz en I344. Realmente es un tratado aristotélico, basado en el ideario de la política de Aristóteles y santo Tomás de Aquino. Originalmente fue redactado a finales del siglo xIII, posiblemente en el contexto de la corte papal de Bonifacio VIII, para la formación del futuro rey de Francia, el infante Felipe IV el Hermoso. Gozó de un gran éxito en las cortes europeas y también en la castellana, a saber, por ejemplo, que el canciller Ayala ya lo cita:

Cuál regimiento deven los príncipes tener es escripto en los libros que solemos leer; Egidio el romano, omne de grant saber, en Regimine prinçipum, lo fue bien conponer ${ }^{15}$.

${ }_{12}$ Ambos son versiones castellanas de un texto que la tradición cristiana tomó a su vez de uno árabe llamado Sirr al'-asrar, atribuido a Yahya ibn al-Batriq. En este caso parece que retoman la tradición de un corpus de consejos que Aristóteles dedicó a Alejandro. Gómez Redondo, F.: op. cit., pp. 274 y ss. Bizzarri, H.: Secreto de los secretos. Poridat de las poridades. Versiones castellanas del Pseudo-Aristóteles Secretum Secretorum, Colección Parnaseo, vol. I2, Universidad de Valencia, 20Io, http://parnaseo.uv.es/Editorial/Parnaseorz/Parnaseoi2.pdf.

${ }_{13}$ Posee una estructura de capítulos muy bien pormenorizada, relativa a valores básicos del príncipe y consejos de buen gobierno. Bizzarri, H.: op. cit., vol. i2, Universidad de Valencia, 20 Io.

${ }^{14}$ La versión editada más completa en BizzarRi, H.: Castigos del rey don Sancho IV, Madrid, $200 I$.

is Gómez Redondo, F.: op. cit., vol. 2, p. 1707. Hay que destacar que la finalidad del texto, según el estudioso Ullmann, W.: Historia del pensamiento político de la Edad Media, p. I2o, 
La versión de García de Castrojeriz traduce el original del latín, manteniendo su estructura en tres libros: el primero sobre las virtudes; el segundo sobre el gobierno de la casa, y el tercero sobre el gobierno de las ciudades y del reino. La obra busca, en primer lugar, promover los valores morales en el monarca, contra los pecados que pueda cometer, en relación a la formación del individuo. Es todo un ejemplo de virtudes del buen creyente, cuajado de consejos espirituales y morales para el buen regimiento del reino. En este caso se añade como novedad también la aparición de buenos príncipes cristianos antiguos a modo de ejemplo para los modernos ${ }^{16}$.

Existen compilaciones de textos medievales que, sin llegar a constituir un tratado específico, tienen como función dotar de exempla a las actitudes de los príncipes, como elementos rectores de vida virtuosa. Este es el caso de los Proverbios o sentencias espirituales y morales ${ }^{17}$, procedentes del manuscrito de El Escorial del mismo nombre. Así, hay un primer capítulo de carácter moral con respecto a las virtudes del monarca, enfocado como casi siempre desde la óptica religiosa. Posteriormente, se continúa con un segundo apartado sobre el regimiento de la ciudad y del reino, como ámbitos de aplicación directos de la virtud regia. En tercer lugar, se trata sobre la tiranía y el tirano, que son calificados negativamente. En general, y aunque sean proverbios, constituyen un conjunto de consejos o frases hechas compilados con un claro enfoque sapiencial.

En un sentido muy parecido se hallan otros conjuntos de proverbios medievales, tales como la Floresta de Filósofos, atribuida a Fernán Pérez de Guzmán, y los Dichos de Séneca en el acto de caballería, a Alfonso de Cartagena. Realmente nos muestran un conjunto de dichos y proverbios, en este caso tomando sentencias de sus diversas lecturas, aunque centrándose en ambos casos en la figura de Séneca, que parece adquirir una relevancia notable en el entorno cortesano en lo referente a su figura política. También se aprecia una mayor preocupación por los hechos de carácter militar y las regulaciones cortesanas, aspectos estos de un interés destacado para la nobleza de la época.

Durante el reinado de Enrique IV, sobre todo en sus últimos años, existió un conjunto de obras que parecen responder a la conflictividad extraordinaria que

«estribaba en demostrar que el papa tenía soberanía (llamada en la obra supremacía) sobre la totalidad del mundo, abarcándolo todo y a todos, y que en consecuencia los príncipes (así como los eclesiásticos) eran súbditos del papa». En definitiva, es un tratado que extiende el concepto de la teocracia pontificia, ya defendida desde tiempos de Inocencio III en el IV Concilio de Letrán de i2I5.

16 Gómez Redondo, F.: op. cit., vol. 2, p. 1713. «E eso mismo leemos de Carlos el Grande, que fue muy estudioso e supo muy bien las artes liberales, e mandolas pintar en su palacio, e aún supo mucho de la Teología e especialmente amaba mucho los libros de San Agustín, el cual le enseñó la Lógica e la Retórica e la Astrología. E fizo pasar el estudio de Roma a París e envió a Roma a aprender el canto, porque non se pagaba de cómo cantaban los franceses, e mandó que enseñasen en toda Francia las maneras del canto de Roma, e fue mucho de alabar en esto».

17 Haro Cortés, M.: «Una nueva colección de sentencias: Proverbios o sentencias espirituales y morales», Revista de Literatura Medieval, I3:I (200I), pp. 9-43. La estructura de las sentencias y su orden, según la autora que lo referencia, responde claramente a la específica de la literatura sapiencial, enfocada a la educación de un futuro rey. 
EL CONCEPTO DE INFANCIA EN LA BAJA EDAD MEDIA EN CASTILLA A TRAVÉS

DE LOS «ESPEJOS DE PRÍNCIPES» (SIGLOS XIII-XV)

ENRIQUE ASENJO TRAVESÍ

abarcaron el periodo de gobierno de este monarca hasta su muerte ${ }^{18}$. El primero de los autores que destacó en estas fechas fue Rodrigo Sánchez de Arévalo, con dos obras significativas como Suma política y Vergel de los príncipes. En ambos casos basa su información en los tratados de Política y Ética de Aristóteles. La diferencia con otros tratados anteriores, al menos en la Suma Política, es que no es un «Espejo de Príncipes» al uso, dado que se ordena más como una reflexión que como un conjunto de consejos para el heredero al trono de un reino cristiano. Además, se elimina de los primeros capítulos la retahíla de principios morales cristianos que veíamos en obras anteriores, comenzando directamente por la fundación y el gobierno de la ciudad. El segundo tratado de la Suma se centra en el regimiento político, como consecuencia derivada de la fundación de la ciudad. Aquí sí aparecen principios morales como rectores de las actuaciones del prínci$\mathrm{pe}^{19}$. Existe una mezcla evidente entre valores humanos y religiosos, pero resulta evidente que se quiere salvaguardar el principio de efectividad del gobierno, aunque se manifieste posteriormente que el rey ha de guardar en primer lugar la ley de Dios, y establecer leyes justas por ellas.

El segundo tratado del Vergel de Príncipes tiene un carácter más profano todavía, dado que se dedica en exclusiva al ocio de los príncipes como un elemento más de su formación. Posiblemente, y dada la posición del autor cercana al bando de Isabel, constituye una rareza y una crítica a las escasas virtudes del monarca, como los estudiosos han deducido ${ }^{20}$. Las tres actividades que trata son la milicia, la caza y la música, defendidas mediante las excelencias que inculcan en el espíritu del príncipe. Entre ellas destacan la «prudencia bélica», la obediencia, la magnanimidad, la paciencia, la perseverancia, la fortaleza, etc. Con respecto a la música, la incluye dentro de las siete artes liberales, y tendría como fin el evitar pecados, puesto que purifica el corazón de malas pasiones.

Otro texto de menor relevancia que los dos anteriores es el anónimo titulado Carta al rey sobre el regimiento de su vivienda ${ }^{21}$. En él se hace una defensa de los principios morales emanados de los valores cristianos como principios rectores del buen príncipe. Es decir, se defiende de nuevo una visión tradicional en la que

18 Las luchas entre bandos nobiliarios que se libraron entre I454 y I474, ya sobrevenidas de época anterior, contribuyeron al florecimiento literario de tratados políticos y de buen gobierno, no tanto por la línea fiel al rey, sino por la alternativa que constituyen el infante don Alfonso primero, y su hermana la infanta Isabel después, ambos hermanos del rey.

19 Gómez Redondo, F.: op. cit., vol. Iv, Madrid, 2007, p. 3617: «Ca debe ser justo, inocente, amigable, piadoso, gracioso, concorde, rigoroso cuanto cumple, umano, verdadero, prudente, bien acordable, inteligente, proveído, circunspecto, enseñable, bien flexible, temperado, continente, clemente, modesto, fuerte, magnánimo, magnífico, liberal, paciente, constante, manso e umilde (ed. JBP, 93; ed. MP, 285 a)».

20 Pérez Priego, M. A.: «Sobre la configuración literaria de los “espejos de príncipes” en el siglo xv castellano», Studia Hispánica Medievalia III, pp. I37-I50, p. I42. Esta temática se basa también en los escritos de pensadores griegos como Platón o Aristóteles, que prestaron argumentos para afirmar que se debía recurrir a determinados elementos de ocio saludable para la deleitación reparadora del alma.

${ }^{21}$ Rodríguez Velasco, J.: «Coordenadas y texto de una carta para regimiento del rey», en Quién hubiese tal ventura. Medieval hispanic studies in honour of Alan Deyermond, London, 1997, pp. 159-168. 
el rey es ante todo buen cristiano, y de su virtud emana el buen gobierno. Aun así no se precisa el medio de formación del rey, sino las tentaciones o pecados que debe evitar, tales como la soberbia, la ignorancia, etc. Se recomienda la adquisición de una sólida disciplina para templar el espíritu y el movimiento del príncipe o del rey, lo cual ayuda a regir a los pueblos ${ }^{22}$.

Uno de los últimos tratadistas de literatura sapiencial del siglo xv fue Pedro de Chinchilla, quien redactó un texto titulado Exortación o ynformaçión de buena e sancta doctrina fecha por Pedro de Chinchilla al muy alto e muy poderoso y esclareçido príncipe y señor don Alfonso, por la graçia de Dios rey de Castilla y León ${ }^{23}$. El texto inicial fue probablemente un regimiento nobiliario readaptado para el príncipe Alfonso, con lo cual tuvo que haber algún tipo de modificación con respecto a la obra inicial que realizó el autor. Dividió su tratado en tres partes: la primera, donde sigue claramente el De regimini principum de Egidio Romano, versa sobre la bienandanza del Príncipe. La segunda, sobre las virtudes que deben regir su vida. La tercera es una miscelánea de consejos y admoniciones. El texto se rige por la adecuación a las cuatro virtudes cardinales que concebía Aristóteles, con un ordenamiento de ideas que se asemeja más a un opúsculo religioso que a un tratado de formación política y buen gobierno.

El último texto relativo a la formación principesca que abarca este periodo es el famoso Doctrinal de Príncipes de Diego de Valera, redactado entre I474 y I477, y dedicado a Fernando de Aragón ${ }^{24}$. En realidad recoge textos y epístolas anteriores, que tienen todos ellos el valor de anteponer las tareas relativas al buen gobierno que el futuro monarca debe realizar, antes que exponer los valores morales de su alma cristiana, como primera de las medidas formativas. Destaca sobre todo en su escrito por procurar que el rey busque una posición ecuánime entre los diversos bandos nobiliarios que habían sumido Castilla en un absoluto caos político y militar, al tiempo que debe rodearse de consejeros fieles, que antepongan el bien del reino al suyo propio, consejo que también aplica al propio príncipe ${ }^{25}$.

22 Gómez Redondo, F.: op. cit., vol. iv, Madrid, 2007, pp. 3637. «Ca el que govierna la tierra ha de usar de grant madureza, porque donde piensa enderesçar non tuerça, ca assí como la ignorancia del que govierna destruye la tierra, assí la prudençia con obra la sana, por lo cual, los que gobiernan los pueblos deven amar la claridat de la sçiençia, ca el gobernador sabio es firmeza de su pueblo».

${ }_{23}$ Analizado por Palacios Martín, B.: «La educación del rey a través de los espejos de príncipe. Un modelo tardomedieval», en L'enseignement religieux dan la Couronne de Castille. Incidences spirituelles et sociales (XIII $-X V^{e}$ siècle), Collection de la Casa de Velázquez, 79, Madrid, 2003, pp. 2942. El texto que nos ocupa se dedicó al infante don Alfonso, hermano del rey Enrique IV, que fue depuesto en efigie por los partidarios de aquel en la farsa de Ávila en ${ }^{4} 465$, momento en el que fue tomado como rey el infante.

${ }_{24}$ Valera, D. de: Espejo de verdadera nobleza, en Penna, M. (ed.): Prosistas castellanos del siglo XV, Madrid, 1959 .

${ }_{25}$ SCandellari, S.: «Mosén Diego de Valera y los consejos de príncipes», Res Publica, i8 (2007), pp. I4I-I62. «Se desprende de la lectura del Doctrinal de príncipes que son muchas las obras que han influido en la formación cultural de Valera y, asimismo, que muchas de éstas aparecen citadas directamente por el autor: dejando al margen su profunda cultura caballeresca la cual le permite escribir unos tratados sobre dicha materia o con ésta relacionada, las fuentes que nos interesan más aquí se refieren a la Biblia, a la enseñanza del Apóstol Pablo, a San Gregorio, San Agustín y San 
EL CONCEPTO DE INFANCIA EN LA BAJA EDAD MEDIA EN CASTILLA A TRAVÉS

DE LOS «ESPEJOS DE PRÍNCIPES» (SIGLOS XIII-XV)

ENRIQUE ASENJO TRAVESÍ

\section{El concepto de infancia a través de la literatura sapiencial en el entorno cortesano de la Castilla bajomedieval}

Durante el periodo medieval, no hay prácticamente información sobre la educación del conjunto de la sociedad, y los estudios sobre la infancia se centran sobre todo en las élites de esta época ${ }^{26}$. Desde el punto de vista estadístico, el valor de la sucesión a través de la infancia viene realzado por los datos, siendo pocos tan elocuentes como que de los reyes castellanos y aragoneses tardomedievales naciesen en su totalidad 80 vástagos y 34 muriesen antes de alcanzar la adolescencia, es decir, un $42,5 \%{ }^{27}$.

La infancia y juventud en un entorno cortesano tenía ventajas con respecto a otros estratos sociales. Por lógica, las condiciones materiales y de vida eran superiores a las de otros grupos. Sin embargo, en función de las circunstancias políticas, un niño o joven podía ser moneda de cambios como rehén garante de un acuerdo o de una paz, como el caso que relata Fernán Pérez de Guzmán en sus Generaciones y Semblazas, relativo a la infancia de Gómez Manrique ${ }^{28}$.

La literatura sapiencial se desarrolla principalmente para la educación de príncipes, pero vamos a tratar de diferenciar, entre los siglos XIII al Xv, qué etapas eran consideradas y de qué modo. En primer lugar, es más que probable que durante este periodo se siguiesen los postulados de diferenciación por edades que ya se conocían de época anterior, bien una infancia tal y como distinguen san Agustín, o incluso san Jerónimo y san Isidoro, hasta los siete años. Después de los festejos habituales y celebraciones posteriores al nacimiento y bautizo del bebé, mayores si este era varón, los niños vivirían durante esta etapa en un mundo femenino, como sucedía en la Antigüedad, siendo cuidados por sus madres y, en el caso de familias nobles o adineradas, por nodrizas ${ }^{29}$. No tenemos constancia para el caso

Isidoro, Santo Tomás y Egidio Romano, Bartolo y a las Partidas, Alfonso de Cartagena, sin olvidar, desde luego a los clásicos que cita en cuantía y entre ellos sobresalen Aristóteles, Cicerón, Lactancio y Séneca», p. 158.

26 Según Beceiro Pita, I.: «La educación: un derecho y un deber del cortesano», en La enseñanza en la Edad Media, X (Semana de Estudios Medievales) (2000), pp. 175-206. "Para comprender esta parquedad documental hay que tener en cuenta, en primer lugar, que los escritos que informan sobre las actividades de los personajes nobles se centran en los acontecimientos de su vida pública que se consideran dignos de ser conservados en la memoria colectiva y, al igual que sigue ocurriendo en la actualidad, éstos arrancan de la juventud. La consecuencia es un relegamiento a segundo plano de la infancia y la adolescencia, que son precisamente las etapas en las que se realiza la labor formativa».

27 Cabrera Sánchez, M.: «La muerte de los niños de sangre real durante el medioevo. Aproximación al tema a través de las crónicas», En la España Medieval, vol. 31, (2008), pp. 217-248, p. 222.

28 Pérez de Guzmán, F.: Generaciones y semblanzas, Londres, 1965, p. 22. Tomado de NorA Arroñada, S.: «Algunas notas sobre la infancia noble en la Baja Edad Media castellana», Historia. Instituciones. Documentos, 34 (2007), pp. 9-27. «Fue dado en rehenes al rey de Granada con otros fijos de cavalleros de Castilla, e como era niño, por enduzimiento e engaño de los moros tornóse moro. Desque fue onbre conoció el error en que biuía e vínose a Castilla e reconcilióse con la fe».

29 Mac Pherson, I. R. y Tate, R. B. (eds.): D. Juan Manuel. Libro de los estados, Madrid, I99I, pp. 196-197. «Otrosi a sus fijos deuelo fazer en esta manera bien en quanto fueren tan niños que non saben fablar nin andar, deuenles catar buenas amas, que sean de la mejor sangre et mas alta et mas 
de la península ibérica del porcentaje de niños que serían criados en esta etapa por amas de cría lejos de su hogar, pero parece que podría ser elevado, al igual que otros lugares de Europa, donde sí está constatado, como el caso de Toscana ${ }^{30}$. Es muy probable que, en entornos privilegiados, los niños se criasen frecuentemente con nodrizas durante sus primeros años para, a partir de los tres o cuatro, regresar al entorno de su familia biológica, o permanecer con amas de cría separados del entorno familiar ${ }^{31}$. Muy poco se conoce del aprendizaje en esta etapa, más que el habla, adoptada probablemente a través del silabeo de las palabras a través de mera imitación por parte del niño. Posteriormente, aprendería un conjunto de normas de comportamiento propias de la sociedad en la que se inserta, a través de la familia, y también de los adultos cercanos, siendo responsables siempre y en primer lugar los padres. Es el conjunto de normas que Rochais denomina conciencia socializada ${ }^{32}$.

Un indicio de la presencia de una etapa específica como la infancia, al menos en el entorno cortesano y nobiliario, sería la existencia de juguetes, que han sido hallados en muy pequeña escala a través de la arqueología. Todos ellos responden a una actividad de juego y ocio propia de la infancia y la niñez. Pero poco más que lo material podemos decir sobre las actividades que desarrollaron ${ }^{33}$, o la percepción positiva o negativa que los tratadistas podían tener sobre las etapas.

Para algunos autores, posteriormente vendría la niñez, hasta los catorce años, y, por último, la adolescencia ${ }^{34}$. La edad adulta era considerada en general desde los I2 años para las niñas y los I4 para los niños. En general se entendía como barrera reproductiva a partir de la cual se entra de lleno en el mundo de los adultos. La mayoría de la sociedad de esta época pasaba rápidamente desde la niñez al mundo de

linda que pudieren aver. Ca çierto es que del padre o de la madre en afuera que non ay ninguna cosa de que los omnes tanto tomen nin a quanto salgan nin a quanto semejen en sus voluntades et en sus obras commo a las amas cuya leche mamaran».

30 García Herrero, M. C.: «Elementos para una historia de la infancia y de la juventud a finales de la Edad Media», en Iglesia Duarte, J. I. de la (coord.): viII Semana de Estudios Medievales: La vida cotidiana en la Edad Media, Nájera, 1997, pp. 223-252, p. 236.

${ }_{31}$ Se sabe que en los entornos reales había toda una pléyade de personal dedicado a la atención de los infantes por parte del personal doméstico de la corte, tales como mozos de espuelas, mozos de ballesta, mozos de plata, criados, mozo de capilla y sirvientes en general que eran recompensados con largueza por los monarcas. También se emplearon instrumentos específicos para su desarrollo físico. Por ejemplo, a la hora de andar había las llamadas «polleras» o andadores, que dejaban libres los brazos de los niños, liberándolos de las fajas con los que se les comprimía desde época de los griegos. En este sentido, resulta muy revelador el testimonio que aporta el cronista F. de Andrada, quien menciona que, cuando el futuro Juan III de Portugal empezó a dar sus primeros pasos, su padre, el rey Manuel el Afortunado, encomendó el niño a un individuo llamado Gonzalo Figueira, con el fin de que acompañase al pequeño y evitase los peligros a los que podía exponerse. También había «mecedoras» como personal para cuidar a los niños en su sueño, y diverso cuerpo de personal que, probablemente, hacían la vida de los niños de la realeza más segura, y constituían en ocasiones una familia más próxima que la de los propios progenitores. CABrera Sánchez, M.: op. cit., pp. 23I-232.

32 Rochais, A.: Las conciencias, Madrid, I984, cap. 2.

33 Alfonso Cabrera, S.: «Juegos y juguetes infantiles en el arte medieval», Revista Digital de Iconografía Medieval, vol. viII, n. ${ }^{\circ}$ I5 (2016), pp. 5I-65.

34 San Isidoro de Sevilla: Etimologías, vol. II, Madrid, Bac, I982, p. 39. 
EL CONCEPTO DE INFANCIA EN LA BAJA EDAD MEDIA EN CASTILLA A TRAVÉS

DE LOS «ESPEJOS DE PRÍNCIPES» (SIGLOS XIII-XV)

ENRIQUE ASENJO TRAVESÍ

los adultos sin ninguna transición. En la Edad Media, hemos de considerar que las etapas formativas, al revés que en el mundo clásico, no estuvieron condicionadas por una serie de actuaciones específicas para formar a los individuos. Esto no quiere decir que no se realicen esfuerzos por educar a los niños y jóvenes, pero es muy probable que estos se concentren en unas élites muy distinguidas, además de que varíen las metodologías en función de las circunstancias y las áreas geográficas.

La nobleza y la realeza, como elementos sociales diferenciados del resto del cuerpo orgánico medieval, tuvieron también etapas formativas propias entre la niñez y la madurez de un estadio adulto. La juventud es un espacio breve de tránsito, en el cual se insertaron los textos de literatura sapiencial. En la etapa que hoy conocemos como adolescencia, el aprendizaje será sustituido por un estilo de educación mucho más formal, en el que los jóvenes deben leer y adquirir unos valores morales a través del intelecto. Sin embargo, este es un mundo reservado solo a los hombres, las mujeres de la Baja Edad Media entran en el concepto de lo que nosotros conocemos como gentiles doncellas. La pureza e inocencia de las niñas guardada en un cuerpo adulto es el mayor bien, reflejado en la iconografía de la dama y el unicornio ${ }^{35}$. Por tanto, debe permanecer custodiada y guardada como un preciado bien. Además, su vida en el exterior debe ser un ejercicio y demostración de virtud cristiana piadosa.

Los jóvenes, sin embargo, eran ruidosos y bulliciosos. Su mundo era el contrario de las mujeres. Se conocen casos de asociaciones de hombres jóvenes que se dedicaban a la danza y a la música, estableciéndose estatutos incluso de las mismas, en los que se regulaban sus actividades. Esto implicaba una regulación obvia de sus acciones, aunque fuese por festividades como los carnavales, la fiesta del obispillo, la fiesta de los locos o la fiesta del asno ${ }^{36}$.

Con respecto al tema que nos ocupa en el estudio, hemos de considerar en primer lugar que, en la literatura castellana relativa al género de «Espejos de Príncipes», no hay una referencia específica a los grupos de edad, aunque se da por supuesto que los textos están dirigidos a los herederos al trono de los Estados medievales, principalmente monarquías. Los antecedentes más remotos de los Specula Principum proceden del mundo carolingio altomedieval ${ }^{37}$.

35 García Herrero, M. C.: op. cit., Nájera, 1997, pp. 244-245. Se advertía a la muchacha de que se cuidase del exceso de curiosidad sobre el mundo y se guardase de exhibirse públicamente. El mundo es entendido como el gran enemigo de la virtud de la joven en la Edad Media y, por tanto, debe alejarse de él para evitar la contaminación de su espíritu.

36 Ladero Quesada, M. Á.: Tópicos y realidades de la Edad Media, vol. 3, Madrid, 2004.

37 Scandellari, S.: op. cit., p. I43. Posteriormente, en la Baja Edad Media, se añadieron una pléyade de consejos prácticos de gobierno para los futuros monarcas, dada la complejidad creciente de los diversos organigramas administrativos y de gobierno de los reinos tardomedievales. Los jóvenes lectores de estos libros y tratados deberían imitar a través del puro exempla que se les mostraba por escrito. El conjunto de autores es muy numeroso, entre los que han influido en la literatura de este género se pueden recordar, empezando por la Via Regis, a Smaragd de Saint-Mihiel, y Sedulio Scoto, Giona d'Orléans, cuya obra De Institia Regia está dedicada a Pipino d'Aquitania; Incmaro de Reims, el poderoso y autoritario obispo; Sedulio Scoto, Alcuino de York, hasta John de Salisbury y el Policraticus, que trata de la diferencia entre el rey y el tirano. 
Dentro de este proceso de formación de los jóvenes príncipes y nobles, los ayos tenían un papel fundamental en la persona de los infantes, tras el mundo femenino de amas de cría en el que habían permanecido hasta ese momento. De hecho, algunos de los ayos nombrados por los reyes fueron los que redactaron algunos de los textos más llamativos de la literatura especular y sapiencial ${ }^{38}$. Es de suponer que los príncipes tendrían una doble formación, con ayos o tutores en las cortes, nombrados por sus padres, que harían las veces de mentores y supervisores de sus acciones. A veces la presencia de estos ayos podía ser benefactora, pero en otras alejaba a los infantes y herederos de la corte, normalmente por situaciones de seguridad de los mismos. Casos conocidos fueron los de Alfonso e Isabel, hermanos menores de Enrique IV, quienes fueron mudados de la casa materna a la corte, por orden de Enrique, unos meses antes de nacer la Beltraneja ${ }^{39}$.

En general, los ayos o tutores serían los encargados de formar intelectual y militarmente al futuro rey. No hay que olvidar que las acciones de los reyes medievales están determinadas tanto por los hechos de armas como por las relaciones cortesanas. Por tanto, el aprendizaje del uso de las armas y el combate era necesario y obligatorio, aunque no tan perentorio como al inicio de la Edad Media. Como complemento y entrenamiento físico de las actividades militares también se practicó el ejercicio de la caza, regulada en la Baja Edad Media a través de tratados de montería y cetrería, relacionados siempre con actividades nobles y propias de la realeza.

La formación intelectual se combinaba con la actividad física a través de las lecturas de autores clásicos, que debían servir de referente. No hay adaptación ni adecuación de las informaciones para los adolescentes y jóvenes, de ningún tipo. Simplemente una selección de textos que ellos deberían leer, para lo cual se asume que desde el siglo XIII se generaliza la alfabetización de las élites dirigentes desde época temprana. Es muy probable que los textos que se utilizasen para la formación de los príncipes fuesen más bien recitados por los tutores y mentores que leídos por ellos mismos. La transmisión de mensajes orales en vez de escritos seguía siendo un canal relativamente frecuente todavía en los siglos XIII y XIV.

${ }_{38}$ «Finalizada la labor del ama de cría, eran los ayos los encargados del cuidado y la educación de los infantes reales. Según las cortes de Palencia debía ser un caballero hidalgo de padre y madre. La lealtad de los ayos hacia los infantes era proverbial, llegando al extremo de cubrir con su cuerpo el del príncipe en plena batalla y muriendo por ello. Ya Alfonso X en sus Partidas dio una serie de normas básicas a ser seguidas en la educación de un príncipe que van desde la higiene hasta la prudencia en el comer y beber y en su comportamiento general. Pero este no fue el único tratado sobre la crianza de los infantes reales; durante la niñez de Alfonso XI, su padre encomendó a Fray Juan García de Castrojeriz que glosara la obra de Egidio Romano sobre el "Regimiento de los príncipes”». Nora Arroñada, S.: «Aproximación a la vida de los niños en la Baja Edad Media española», Meridies (Iv), 1997, pp. 57-70, p. 60.

39 En opinión de Fernán Pérez de Guzmán, y refiriéndose al condestable de Castilla en tiempos de Enrique IV, Ruy López Dávalos, dice: «Porque comunmente los reyes desque son onbres desaman a los que cuando niños los apoderaron, fue asi apartado del monarca e puesto en grande indignacion suya». E incluso hablando de los hijos de Fernando de Antequera comenta «eran muy moços e tocados de aquella dolencia real que es comun e general a todos los reales moços que son regidos e gouernados por ayos e maestros e algunos son que nunca desta dolencia sanan». Nora ArroÑadA, S: op. cit., p. 6r. 
EL CONCEPTO DE INFANCIA EN LA BAJA EDAD MEDIA EN CASTILLA A TRAVÉS

DE LOS «ESPEJOS DE PRÍNCIPES» (SIGLOS XIII-XV)

ENRIQUE ASENJO TRAVESÍ

Por tanto, tenemos un equilibrio entre formación teórica y acción en la educación del joven príncipe, al que se añade la instrucción religiosa. El peso de la Iglesia, y sobre todo el control de la moral de la sociedad que se ejercía sobre la época, hizo que, salvo en contadas excepciones, la formación en los valores propios de la fe cristiana fuese el elemento más destacado de la educación de los príncipes y nobles. Solo al final de la Edad Media aparece un conjunto de saberes prácticos y consejos de buen gobierno como elemento fundamental de los manuales del tipo Spéculo. Todos ellos están enfocados a un gobierno que debe estar caracterizado por la astucia y la sabiduría, en un contexto cambiante e inestable, y con una burocracia creciente. En la medida en que la complejidad de la administración del reino se incremente, aparecerá también de nuevo la influencia clásica bajo la forma del pensamiento de Platón y Aristóteles.

\section{Bibliografía}

Alfonso Cabrera, S.: «Juegos y juguetes infantiles en el arte medieval», Revista Digital de Iconografía Medieval, vol. viII, n. ${ }^{\circ}$ is (20I6), pp. 5I-65.

Beceiro Pita, I.: «La educación: un derecho y un deber del cortesano», La enseñanza en la Edad Media, X Semana de Estudios Medievales, Nájera, 2000, pp. 175-206.

Bizzarri, H.: Flores de filosofía, Ms. escur. S.II.I3, Seminario de Edición y Crítica Textual (I5IO-1997), http://parnaseo.uv.es/Memorabilia/Floresı.html.

Bizzarri, H.: Castigos del rey don Sancho IV, Madrid, 20oI.

Bizzarri, H.: Secreto de los secretos. Poridat de las poridades. Versiones castellanas del PsendoAristóteles Secretum Secretorum, Colección Parnaseo, vol. I2, Universidad de Valencia, 20IO, http://parnaseo.uv.es/Editorial/Parnaseor2/Parnaseor2.pdf

Casado Rigalt, D.: Historia de la educación durante la Antigüedad y la Edad Media, Madrid, UDIMA, 20I5.

FlandRIn, J. L.: «Lugares comunes, tradicionales y modernos, sobre el niño en la familia», en La moral sexual en Occidente, Barcelona, 1984.

García Herrero, M. C.: Las mujeres en Zaragoza en el siglo XV, Zaragoza, 1990.

García Herrero, M. C.: «Elementos para una historia de la infancia y de la juventud a finales de la Edad Media", en Iglesia Duarte, J. I. de la (coord.): viII Semana de Estudios Medievales: La vida cotidiana en la Edad Media, Nájera, 1997, pp. 223-252.

Giménez Soler, A.: «Retrato histórico de la reina doña María», Boletín de la Real Academia de Buenas Letras de Barcelona, I901, pp. 72-8I.

Gómez Redondo, F.: Historia de la prosa medieval castellana, vols. I, II, III y IV, Madrid, I998.

Haro Cortés, M.: «Una nueva colección de sentencias: Proverbios o sentencias espirituales y morales», Revista de Literatura Medieval, I3:I (200I), pp. 9-43.

Ladero Quesada, M. Á.: Tópicos y realidades de la Edad Media, vol. 3, Madrid, 2004.

LANGER, W.: Historia de la infancia, Boston, 1973.

Lucía Megías, J. M.: Flores de filosofía. Transcripción semipaleográfica del ms. 9428 de la Biblioteca Nacional de Madrid (ff. I-I8), Seminario de Edición y Crítica Textual (I5-IO-I997), http://parnaseo.uv.es/Memorabilia/Flores2.html.

Mac Pherson, I. R. y Tate, R. B. (eds.): D. Juan Manuel. Libro de los estados, Madrid, i99i.

Nogales Rincón, D.: «Los espejos de príncipes en Castilla (siglos XIII-Xv): un ejemplo literario de la Castilla Bajomedieval», Medievalismo, 16 (2006), pp. 9-39.

Nora Arroñada, S.: «Aproximación a la vida de los niños en la Baja Edad Media española», Meridies (IV), I997, pp. 57-70. 
Nora Arroñada, S.: «Algunas notas sobre la infancia noble en la Baja Edad Media castellana», Historia. Instituciones. Documentos, 34 (2007), pp. 9-27.

Palacios Martín, B.: «La educación del rey a través de los espejos de príncipe. Un modelo tardomedieval», en L'enseignement religieux dan la Couronne de Castille. Incidences spirituelles et sociales (XIII $-X V^{e}$ siècle), Collection de la Casa de Velázquez, 79, Madrid, 2003, pp. 29-42.

PÉREZ de GuzMán, F.: Generaciones y semblanzas, London, 1965.

Pérez Priego, M. A.: «Sobre la configuración literaria de los “espejos de príncipes” en el siglo xv castellano", Studia Hispánica Medievalia III, pp. I37-I50.

Rochais, A.: Las conciencias, Madrid, i984.

Rodríguez Velasco, J.: «Coordenadas y texto de una carta para regimiento del rey», en Quién hubiese tal ventura. Medieval Hispanic studies in honour of Alan Deyermond, London, 1997, pp. 159-I68.

SAN Isidoro de Sevilla: Etimologías, vol. II, Madrid. BAC, 1982.

Scandellari, S.: «Mosén Diego de Valera y los consejos de príncipes», Res Publica, i8 (2007), pP. I4I-I62.

Valera, D. de: «Espejo de verdadera nobleza», en Penna, M. (ed.): Prosistas castellanos del siglo XV, Madrid, 1959 .

Walsh, J. K.: El libro de los doze sabios, Madrid, 1975. 